\title{
SÉDIMENTOLOGIE ET DATATION DES DÉPÔTS FLUVIO-ÉOLIENS DU PLÉNIGLACIAIRE WEICHSELIEN À LILLE (VALLÉE DE LA DEÛLE, BASSIN DE L'ESCAUT, FRANCE)
}

\author{
Laurent DESCHODT ${ }^{1}$
}

\begin{abstract}
RÉSUMÉ
Dans la région de Lille, la Deûle a nettement incisé le substrat crayeux. L'incision la plus ancienne se situe sous le bas de versant du Pays de Weppes et est antérieure au Weichselien. Le tracé a été actif jusqu'au Début Glaciaire. Une incision plus récente, antérieure au Pléniglaciaire supérieur weichselien, s'individualise environ un kilomètre plus à l'est. Le chantier de la rue Hegel permet une observation directe d'une partie du remblaiement du chenal pléniglaciaire weichselien. Les alluvions sableuses se développent sur 12,5 mètres, elles se déposent en petits chenaux et sont accompagnées de quelques marqueurs de froids. Elles sont datées de la fin du Pléniglaciaire $(17960 \pm 90 \mathrm{BP}$, soit $21639 \pm 361 \mathrm{cal}$. BP). Elles cèdent la place à des formations fluvio-éoliennes, apparemment en continuité, qui, d'abord litées et argileuses, deviennent lœssiques. A la lumière de la date ${ }^{14} \mathrm{C}$ et de deux datations OSL $(21,7 \pm 1,9$ et $23,7 \pm 2,0 \mathrm{ka})$, la formation limoneuse à dominante éolienne semble se mettre en place rapidement (vers $21,7 \mathrm{ka}$ ?). Ces premières dates effectuées sur le remblaiement lœssique terminal des fonds de vallées du nord de la France sont à considérer avec prudence. Elles impliquent la simultanéité du dépôt éolien en fond de vallée et sur versant à la fin du Pléniglaciaire, plus précocement que dans les vallées sableuses du bassin belgo-néerlandais.
\end{abstract}

Mots-Clés : Deûle, Lille, Weichselien, Pléniglaciaire, alluvions, dépôt fluvio-éolien, lœss, fond de vallée

\section{ABSTRACT}

SEDIMENTOLOGY AND DATING OF WEICHSELIAN UPPER PLENIGLACIAL FLUVIO-AEOLIAN DEPOSITS IN LILLE (DEULE VALLEY, SCHELDE BASIN, FRANCE)

The Deûle River has scoured the chalk substratum in two places in the vicinity of Lille. The older scouring is under the lower slopes of the Weppes country. It occurred prior to the Weichselian. It was still active in the Early Glacial. A more recent scouring, predating the Upper Pleniglacial, appeared one kilometre eastward. The Hegel street excavations allow direct observation of a portion of the in-filled channel; a 12,5 m thick sandy alluvium deposited in small channels associated with indications of cold. It dated to about the end of the Pleniglacial $(17960 \pm 90$ BP, i.e. $21639 \pm 361$ cal. BP). These deposits gave way to -apparently continuous- fluvio-aeolian formations, bedded and clayey at first, becoming loess-like in the upper strata. According to the $\mathrm{C}^{14}$ date and to two OSL dates $(21.7 \pm 1.9$ and $23.7 \pm 2,0 \mathrm{ka})$, this silty, primarily aeolian formation seems to have been rapidly deposited (about $21.7 \mathrm{ka}$ ?). These first dates of loess upper valley infilling from northern France should be considered with caution. They appear to indicate the simultaneity of aeolian deposit on slope and valley bottoms at the end of the Pleniglacial, earlier than in the sandy valleys of Belgium and Netherlands.

Keywords: Deûle River, Lille, Weichselian, Pleniglacial, alluviums, fluvio-aeolian deposits, loess, valley bottom

\section{1 - CONTEXTE ET MÉTHODE}

En 2005, la Communauté Urbaine de Lille a réalisé un bassin de rétention des eaux pluviales à Lille-Lomme, à proximité du canal de la Deûle et de la rue Hegel. L'ouvrage correspond à un bassin cylindrique d'une trentaine de mètres de diamètre et d'une quinzaine de mètres de profondeur. Il est situé en rive gauche de la zone alluvionnaire holocène, au pied du long versant lœssique en pente douce du pays de Weppes (fig. 1 et 2). A cet endroit, les sédiments quaternaires ont 18 mètres d'épaisseur et reposent sur la craie banche sénonienne.

Sur prescription du service régional de l'archéologie, des sondages archéologiques ont été entrepris à l'emplacement du futur bassin. Réalisés en puits à la pelle mécanique sur environ 6 mètres de profondeur, les sondages archéologiques ont permis de repérer le passage de limon (en surface) à des alluvions sableuses. Limitées en profondeur, ces observations ont été complétées par une surveillance de travaux. En accord avec la Communauté Urbaine, nous avons effectué plusieurs levés des sédiments lors du décaissement à l'intérieur de la paroi moulée.

${ }^{1}$ INRAP (Institut National de Recherches Archéologiques Préventives), UMR 8591 et visiteur UMR 8164, Z.I. de la Pilaterie,

11 rue des Champs, F-59650 VILLENEUVE D’ASCQ. Courriel: laurent.deschodt@inrap.fr 

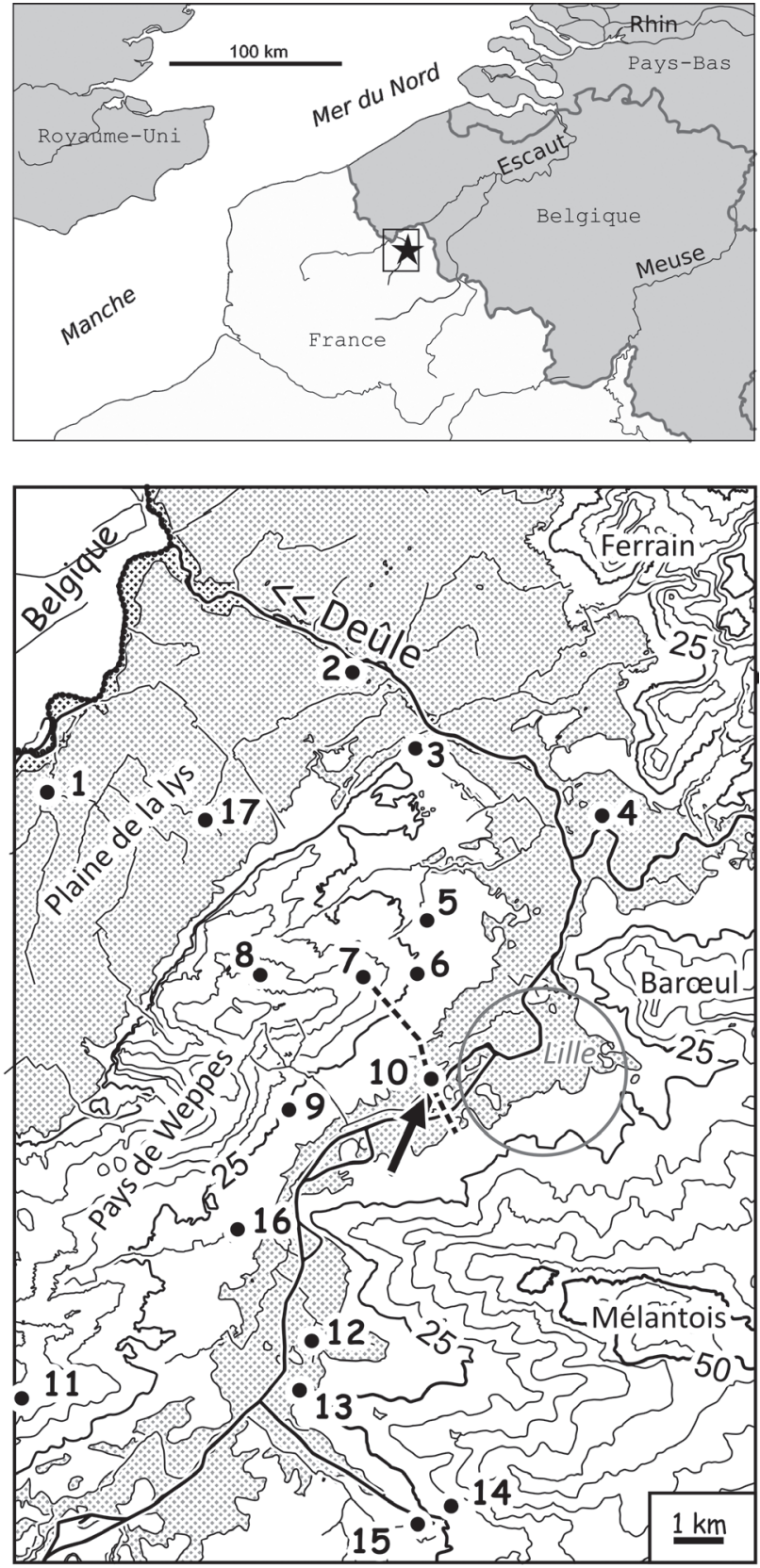

Fig. 1: Localisation du bassin de la rue Hegel dans la vallée de la Deûle et d'observations effectuées dans la région de Lille.

Fond de plan d'après C. Quérel (INRAP, inédit), modifié. Courbes de niveau à équidistance de $5 \mathrm{~m}$ NGF Lallemand. Zone gris clair: entre 15 et $20 \mathrm{~m}$ d'altitude, zone gris-foncé: moins de $15 \mathrm{~m}$ d'altitude. Flèche: Lille-Lomme, bassin de la rue Hegel. Trait interrompu: transect présenté figure 7. Observations (liste non exhaustive): 1/ Houplines (Salvador et al., 2005), 2/ Quesnoy-sur-Deûle (Sommé, 1977), 3/ Wambrechies «Fort du Vert-Galant» (Ladrière, 1883, 1885), 4/ Marquette-lez-Lille «Le Haut-Touquet» (Cercy, 2004), 5/ Lambersart «Les Conquérants», 6/ Lambersart «Briqueterie» (Sommé 1968, 1977), 7/ Lomme (Sommé, 1977), 8/ Prémesques (Sommé, 1977), 9/ Sequedin (Sommé, 1977), 10/ Lille, rue Hegel (Deschodt et al. 2008), 11/ Sainghin-en-Weppes (Sommé, 1977), 12/ Houplin-Ancoisne «Station d'épuration» (Deschodt et al. 2004), 13/ Houplin-Ancoisne «Le Marais de Santes» (Praud et al., 2007; Deschodt et al., 2008), 14/ Seclin «Usine» (Tuffreau et al. 1994), 15/ Seclin «Hôpital» (Deschodt et al. 2008), 16/ Hallennes-lez-Haubourdin (inédit), 17/ Houplines «Le Chemin de l'Aventure» (inédit), 18/ Wavrin (inédit), 19/ Loos «Bassin Joffre» (inédit), 20/ Santes (inédit), 21/ Quesnoy-sur-Deûle «station d'épuration» (inédit).

Fig. 1: Hegel street basin location and other observations in Lille's area. Base map from C. Ouérel (INRAP), modified. Contour lines (interval $5 \mathrm{~m}$ ), NGF Lallemand survey. Light grey area: 15 to 20 meters height, dark grey area: down 15 meters height. Arrow: Hegel street basin. Dashed line: cross-section presented on figure 7. Other observations (partial list) numbered from 1 to 21 (see above).

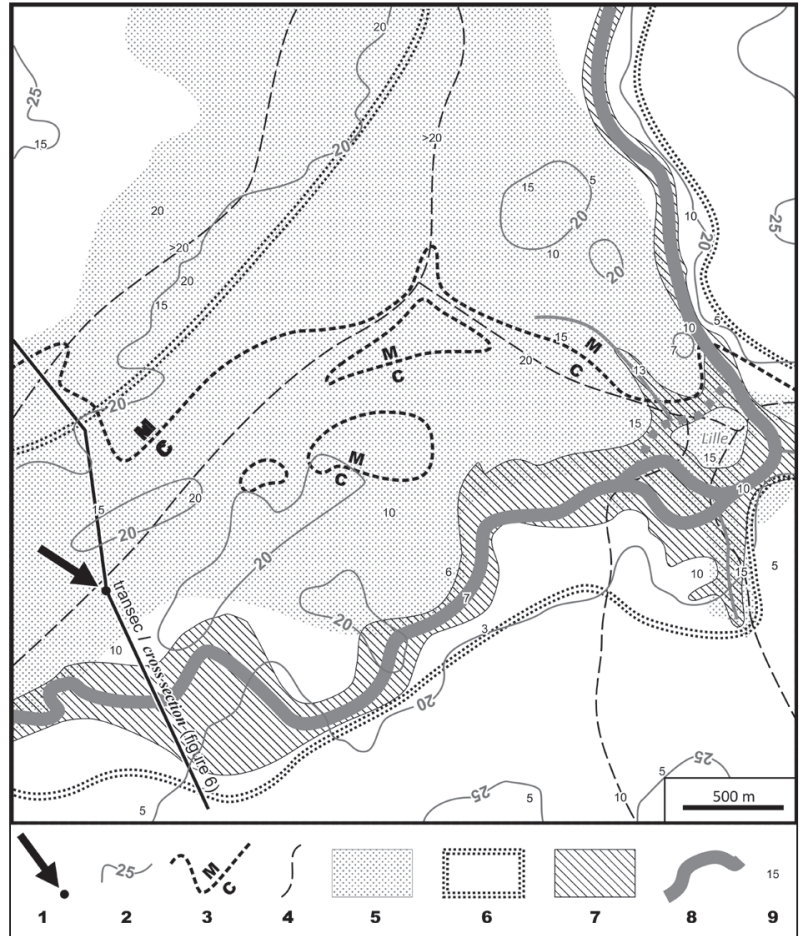

Fig. 2 : Localisation du bassin "rue Hegel" sur une carte des formations superficielles, d'après Deschodt (1999) modifié.

1/ localisation (centre bassin $\left.50^{\circ} 37^{\prime} 54,27^{\prime} \mathrm{N}, 3^{\circ} 01^{\prime} 16,29^{\prime \prime} \mathrm{E}\right), 2 /$ courbe de niveau en mètres NGF Lallemand, d'après la carte IGN 1/25 $000^{\mathrm{e}}, 3$ / limite des affleurements des terrains mésozoïques (M, argile et sable) et cénozoïques ( $C$, craie) sous les dépôts quaternaires, 4/ talweg inscrit dans le substrat ante-quaternaire, 5/ remblaiement quaternaire (antérieure au Pléniglaciaire supérieur), essentiellement sableux, 6/ faisceau du dépôt des formations lœssiques litées du Pléniglaciaire supérieure (lœss remanié par les eaux), $7 /$ dépôts alluviaux attribuables au Tardiglaciaire-Holocène (gravier, sable, limon, tourbe), 8/ lit mineur d'âge tardiglaciaire-holocène probable, 9/ épaisseur approximative des formations sédimentaires attribuables au Quaternaire.

Fig. 2: "Rue Hegel" basin location over a superficial formation map, according to Deschodt (1999) modified. 1/ location (basin center $50^{\circ} 37^{\prime} 54,27^{\prime \prime} N$, 301'16,29'E), 2/ NGF Lallemand survey courtour line, according to the IGN 1/25000 map, 2/ Mesozoïc rocks (M, clay and sand) and Cenozoïc (C, chalk) outcrops limits, concealed by quaternary deposits, 3/ talweg scoured in the ante-quaternary rocks, 4/ meanly sandy previous Weichselian Upper Pleniglacial quaternary infilling, 5/ Weichselian Upper Pleniglacial bedded loessic formations area (water reworked loess), 6/ alluviums attribuable to LateglacialHolocene (gravel, sand, loam, peat), 7/ mean water likely of Lateglacial-Holocene age, 8/ Deûle canal, 9/ Quaternary deposits rough thickness.

\section{2 - OBSERVATIONS}

La surveillance de travaux a permis l'observation en coupe et le levé de la stratigraphie des formations sédimentaires sur de petits témoins totalisant 9 mètres de profondeur (photos 1 à 3). Des chenaux de quelques mètres de larges ont été observés sur des lambeaux accrochés à la paroi (fig. 3). Le mode d'observation implique que les structures sédimentaires de plus grande envergure (éventuels larges chenaux) ne sont pas perçues. Les observations sont détaillées sur la figure 4 et synthétisées sur la figure 5. Nous avons pu effectuer trois datations: deux OSL (Luminescence Optiquement Stimulée) sur sédiment et une ${ }^{14} \mathrm{C}$ (Radiocarbone) sur un bois flotté. Les trois dates confirment un dépôt au cours de la fin du Pléniglaciaire supérieur weichselien. 


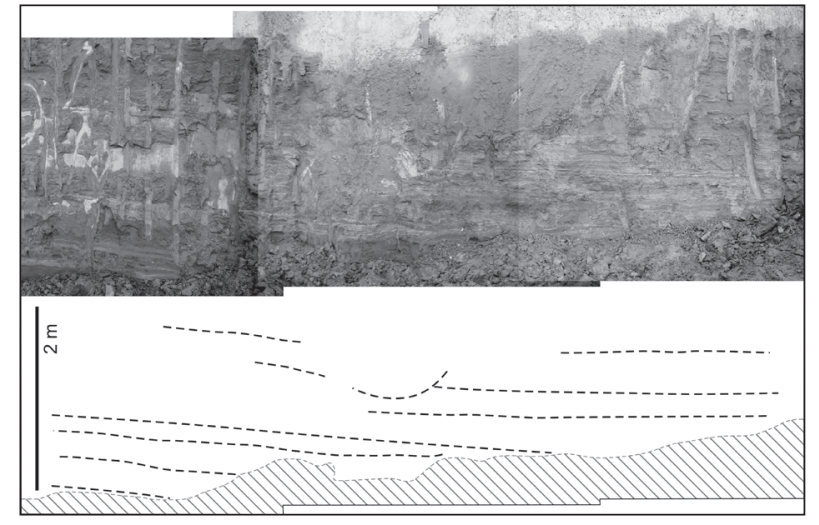

Fig. 3 : Coupe 3, photomontage.

Des lambeaux de terrains contre la paroi permettent de percevoir les structures fluviatiles à moyenne échelle.

Fig. 3: Cross-section 3, photomontage. Earth strips against the wall enable to see medium scale fluvial structures.

Au-dessus de la craie sénonienne, observée dans les sondages géotechniques à 18 mètres de profondeur, nous pouvons synthétiser les sédiments quaternaires en deux formations l'une sableuse (plus de 12 mètres d'épaisseur), l'autre à dominante limoneuse (5,5 mètres d'épaisseur) (fig. 5).

\section{1 - FORMATION SABLEUSE}

\subsection{1 - Membre A: dépôts fluviatiles en larges} chenaux

Le membre $\mathrm{A}$, observé de 9 à $6 \mathrm{~m}$ de profondeur est constitué de dépôts fluviatiles lités, majoritairement sableux mais avec une part limoneuse localement importante. Les lits limoneux peuvent être très clairs, presque blancs, et se caractérisent par une concentration en carbonate (photos 2 et 4 à 6). Les dépôts s'accumulent dans des chenaux (plusieurs mètres de larges, visibles à moyenne échelle; fig. 3). La base du remplissage est généralement tapissée de graviers de craie. Les dépôts sont fréquemment granoclassés positivement. Un galet de craie isolé (environ $5 \mathrm{~cm}, \mathrm{n}^{\circ} 7$, coupe 3 ; fig. 4) témoigne d'un transport par radeau de glace. Une fente à remplissage minéral (sand wedge) est présente, tronquée, au sein des alluvions sableuses. Quelques petits débris végétaux flottés sont associés aux alluvions. L'un d'eux a été daté à $17960 \pm 90$ BP (Lyon-3910, GrA) à 8,7 m de profondeur (21 $639 \pm 361$ cal. BP, 1 sigma, calibrée avec CalCurve: CalPal_2007_HULU, Weninger \& Jöris, 2008).

De petits comblements limoneux lités en fin de cycle sont probablement liés à des écoulements reliques à

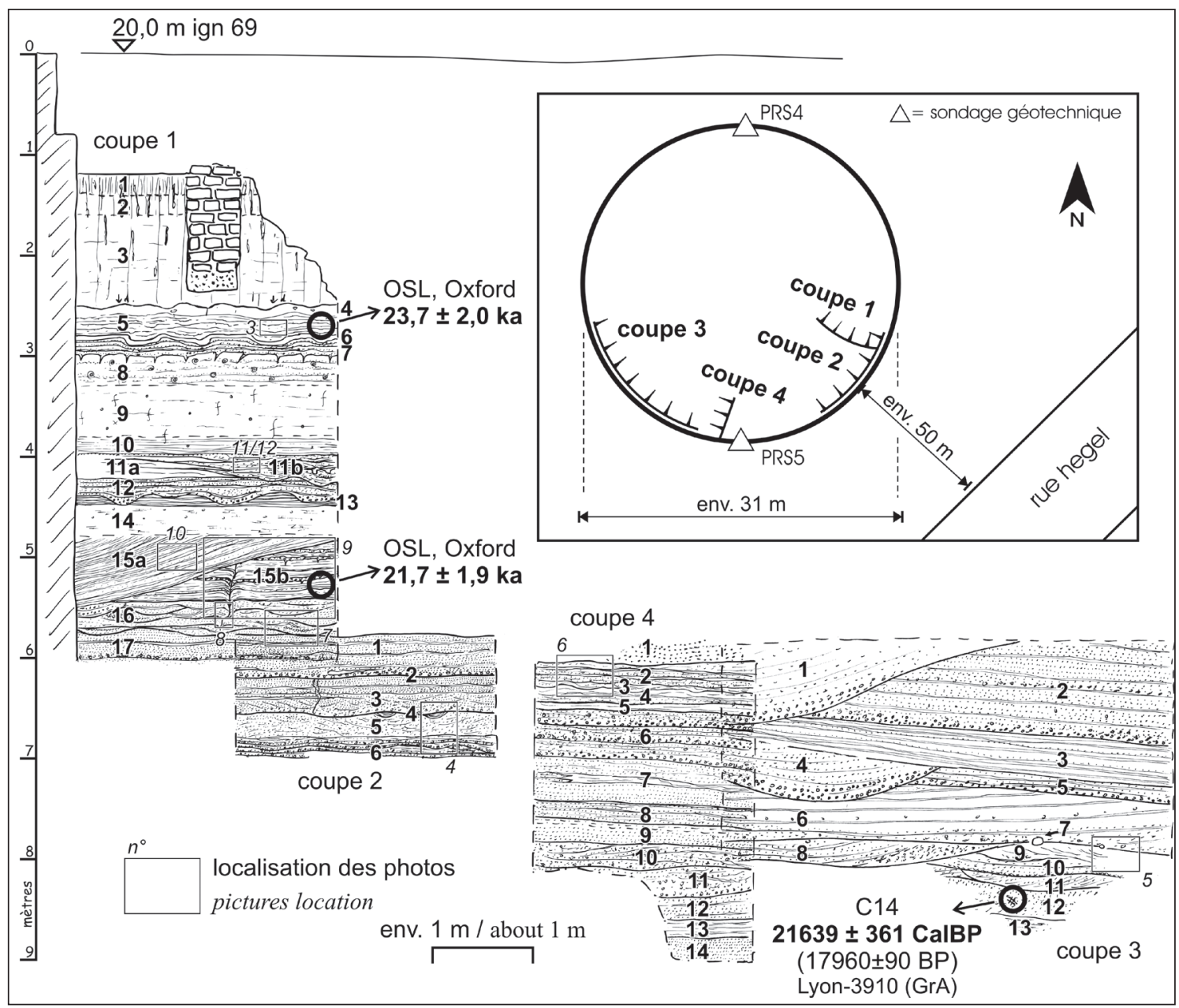

Fig. 4 : Bassin rue Hegel : détail des observations.

Cartouche: localisation schématique des coupes. Description: voir tableau 1.

Fig. 4: Hegel street basin: observation details. Frame: cross-section schematic location. Description: see table 1. 


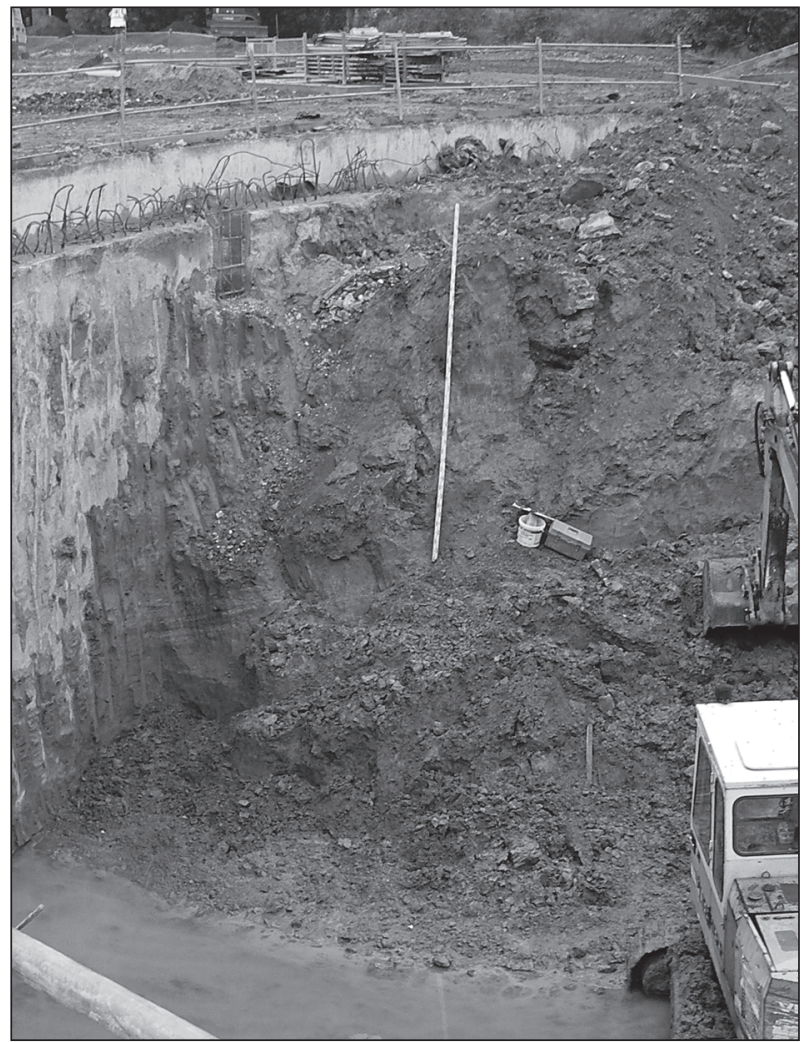

Photo 1 : Vue générale de la coupe 1.

Observation effectuée sur la rampe d'accès.

Photo 1: Cross-section 1 overview. Observation done from the slope.

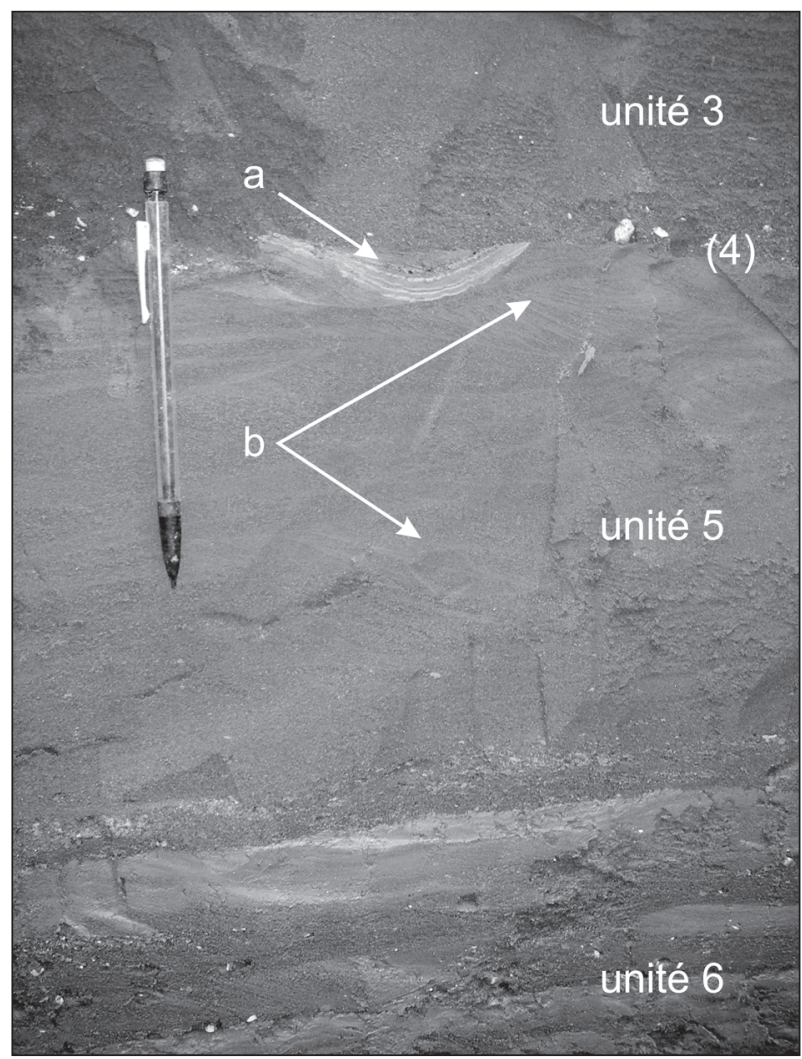

Photo 4 : Coupe 2, unités 3 à 6.

(a) micro-chenal au sommet de 3 , comblés par des litages très fins de limon argileux et de quelques lamines sableuses (fin d'écoulement actif), l'ensemble est tronqué par la limite érosive 4. (b) lamines sablolimoneuses entrecroisées au sein de l'unité 5 .

Photo 4: Cross-section 2, units 3 to 6. (a) micro-channel at 3 top, filled by very fine clayed loamy beds and few sandy beds (end of the flowing), the whole is truncated by the erosive limit 4. (b) cross sandy loamy laminations in the unit 5 .

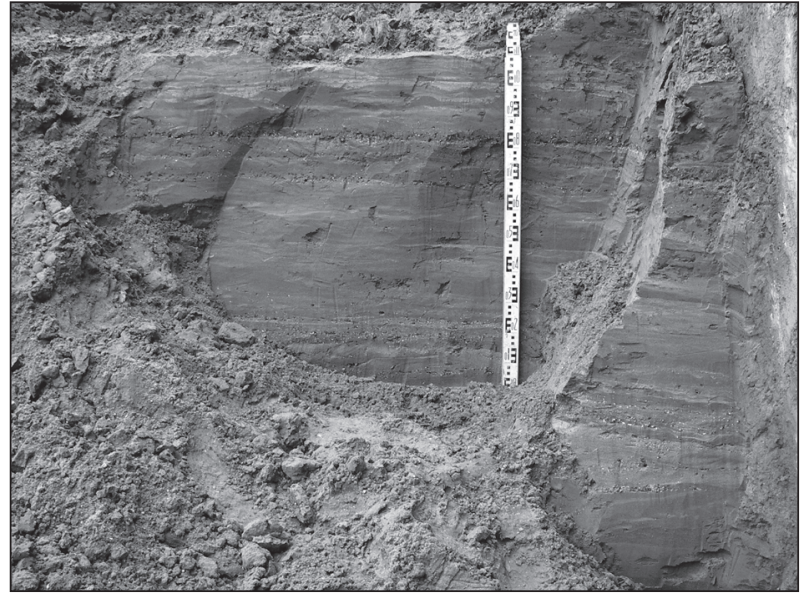

Photo 2 : Coupe 4, vue générale.

Les conditions d'observations mettent surtout en évidence les structures fluviatiles à grande échelle.

Photo 2: Cross-section 4, overview. The observation conditions especially highlight the large-scale fluvial structures.

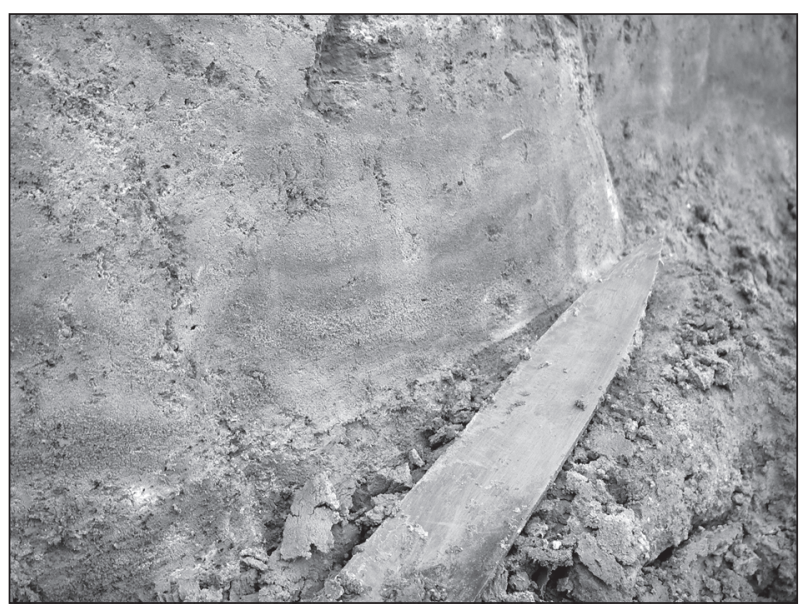

Photo 3: Coupe 1, détail de l'unité 5.

Litages discrets de sablon limoneux dans le limon.

Photo 3: Cross-section 1, unit 5 detail. Discreet loamy sand beds in the loam.

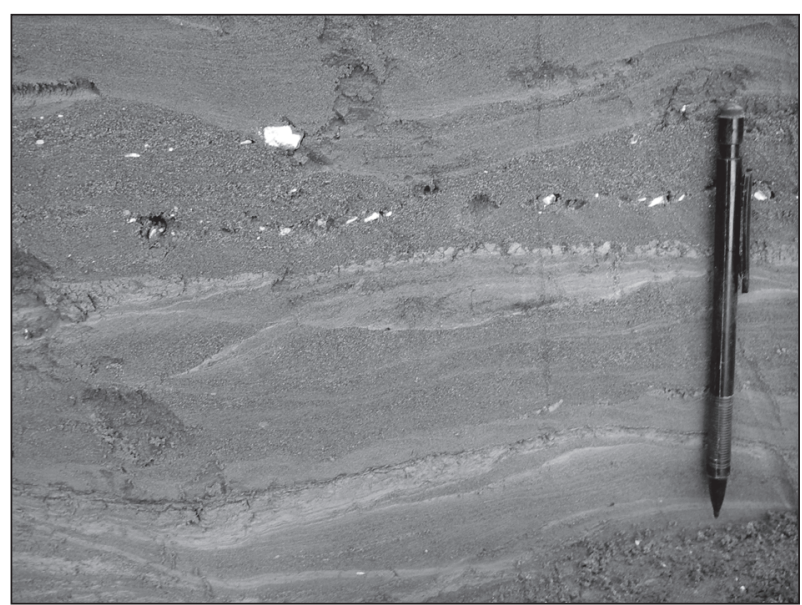

Photo 5: Coupe 3, unités 7 à 11.

Lits de sable, de limon, de gravier, et quelques lamines plus sombres composées de matière organique.

Photo 5: Cross-section 3, units 7 to 11. Sand, loam and gravel beds, and a few darker laminae with organic matter.

la fin de la saison hydrologiquement active (photo 4). De petits tressages (de l'ordre de $0,4 \mathrm{~m}$ de large pour environ $0,1 \mathrm{~m}$ de profondeur sont sporadiquement observés) (photo 4). 
Le style fluvial est difficile à déterminer avec certitude en raison de la perception fragmentée de la géométrie des structures. Les interstratifications sur de faibles distances perçues sur l'observation 3 (unités de détail 8 à 11, coupe 3, fig. 4) ou l'incision par des chenaux de taille apparemment modérée (unités de détail 1 et 4 , coupe 3 ; fig. 4) sont minoritaires par rapport aux structures plus vastes dont nous ne percevons pas les limites. Sans évidence forte, nous pensons être en présence de chenaux méandriformes qui se recoupent dans une plaine à pente longitudinale faible envahie par une charge fine (sable et limon). Le même style attribué à une activité fluviatile contemporaine a été mis en évidence sur la base d'une morphologie héritée, quelques kilomètres plus en amont (à Houplin-Ancoisne, chenal de l'ordre de 20 à 30 mètres de large et rayon de courbure d'environ 50 à 70 mètres; Praud et al., 2007) et dans la plaine voisine de la Scarpe à Coutiches, Vred et Saint-Amand-les-Eaux (chenaux peu profonds jusqu'à une centaine de mètres de large; Deschodt et al., 2012). Dans cette hypothèse, la présence des lits graveleux correspondrait à la charge de fond des chenaux. Les petites structures entrecroisées (unités de détail 5 , coupe 2 sur la figure 4 , photo 4 ) pourraient être l'amorce de petites barres de méandre.

Les observations géotechniques proches (cf. fig. 5 et infra) permettent d'envisager la base du membre A directement sur la craie.

\subsection{2 - Membre B: dépôts fluviatiles en petits chenaux}

Le membre $B$ est peu épais (quelques décimètres) et de faciès proche du membre 1 auquel il succède. Il s'en distingue par une généralisation de petits chenaux tressés (petits chenaux visibles à grande échelle; photo 7), avec un granoclassement bien marqué (un dépôt limoneux argileux souligne la fin des cycles) et la présence d'une fente de cryodessiccation syngénétique (photo 8) qui se prolonge dans le membre $\mathrm{C}$.

\section{2 - FORMATION À DOMINANTE LIMONEUSE}

\subsection{1 - Membre C : dépôts fluviatiles sablo-argileux}

Le membre $\mathrm{C}$ (environ 1 mètre d'épaisseur) est constitué de lamines particulièrement fines et régulières d'argile limoneuse claire alternant avec quelques lamines sableuses (photos 9 et 10). Une discordance interne (probablement le bord d'un petit chenal) sépare une partie inférieure plus sableuse (unité 15A sur la figure 4 où les litages sub-horizontaux sont en continuité avec le membre B sous-jacent) d'une partie plus argileuse (dont les lits ont un pendage marqués, unité 15B sur la figure 4).

L'impression de continuité entre le membre B et la première partie du membre $\mathrm{C}$ est renforcée par la prolongation de la fente de dessiccation syngénétique sousjacente qui prend naissance dans le membre $\mathrm{B}$.

La première partie du membre $\mathrm{C}$ est datée par OSL à $21,7 \pm 1,9 \mathrm{ka}$.

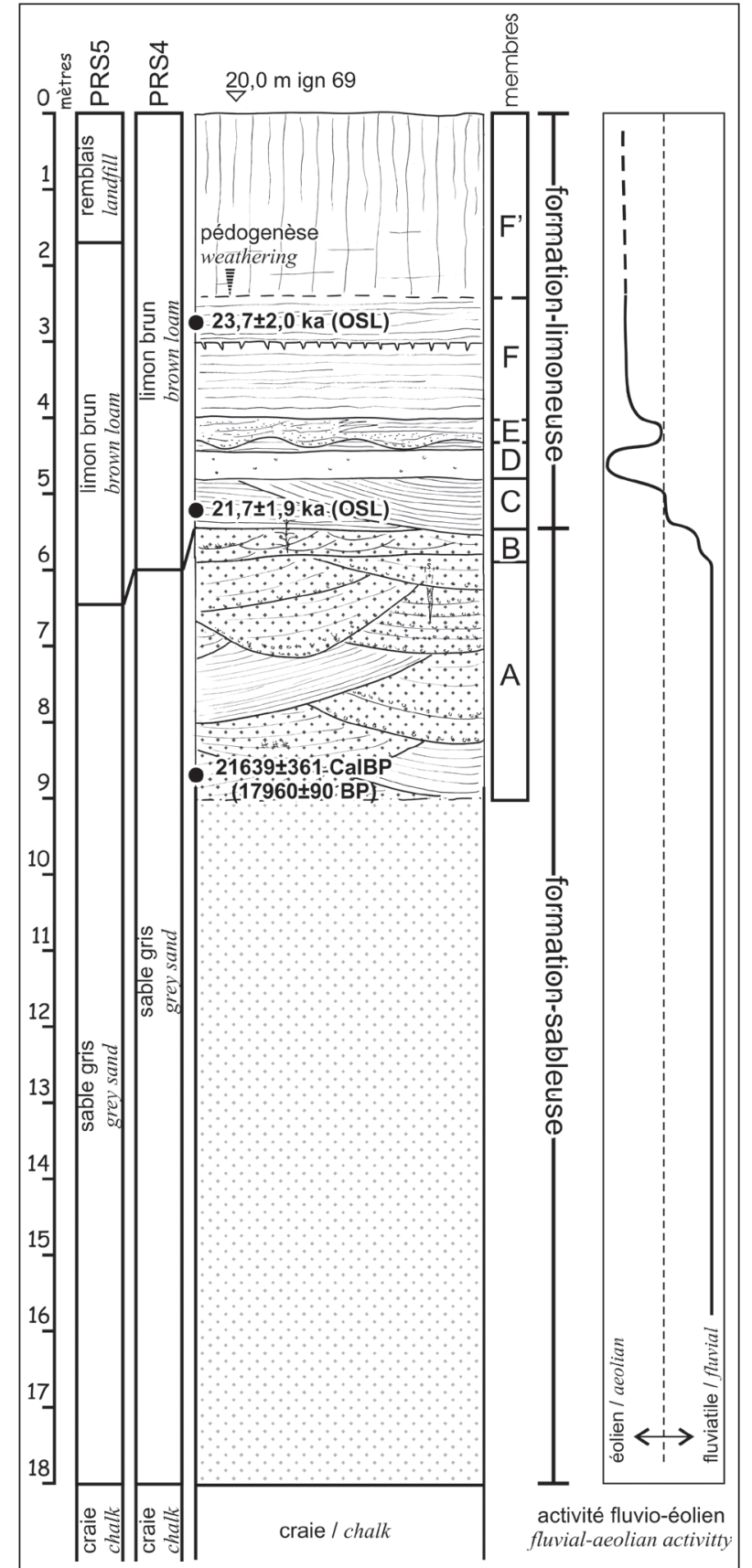

Fig. 5 : Synthèse des observations.

Fig. 5: Observation synthesis.

\subsection{2 - Membre D : limon à graviers de craie}

Le membre $\mathrm{D}$, épais de quelques décimètres, est constitué d'un limon à petits graviers de craie épars et à structure cryogénique (agrégats lamellaires fins). Le sommet du membre est marqué par un retour à des litages argileux très fins et réguliers similaires à ceux du membre $\mathrm{C}$.

\subsection{3 - Membre E : Sable, sablon limoneux et sable lité}

La base du membre $E$ est marquée par une érosion fluviatile. Des sables et graviers reposent en discordance sur le membre D. La majeure partie du membre E est constituée d'un limon ou d'un sablon lité avec lits fins argileux (photo 11). Des agrégats argileux plastiques sont fréquemment repris par les écoulements (photo 12). 


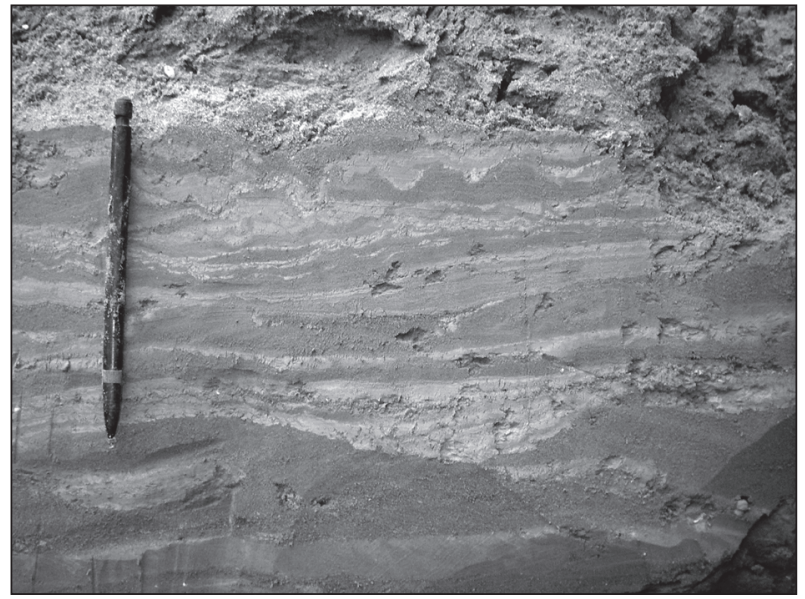

Photo 6: Coupe 4, partie supérieure.

Sous les sable grossiers de l'unité 1 , alternance de limon très fin calcaire, de sable, présence de figures de charge.

Photo 6: Cross-section 4, upper part. Below unit 1 coarse grained sand, fine and very calcareous loam, sand; load casts.

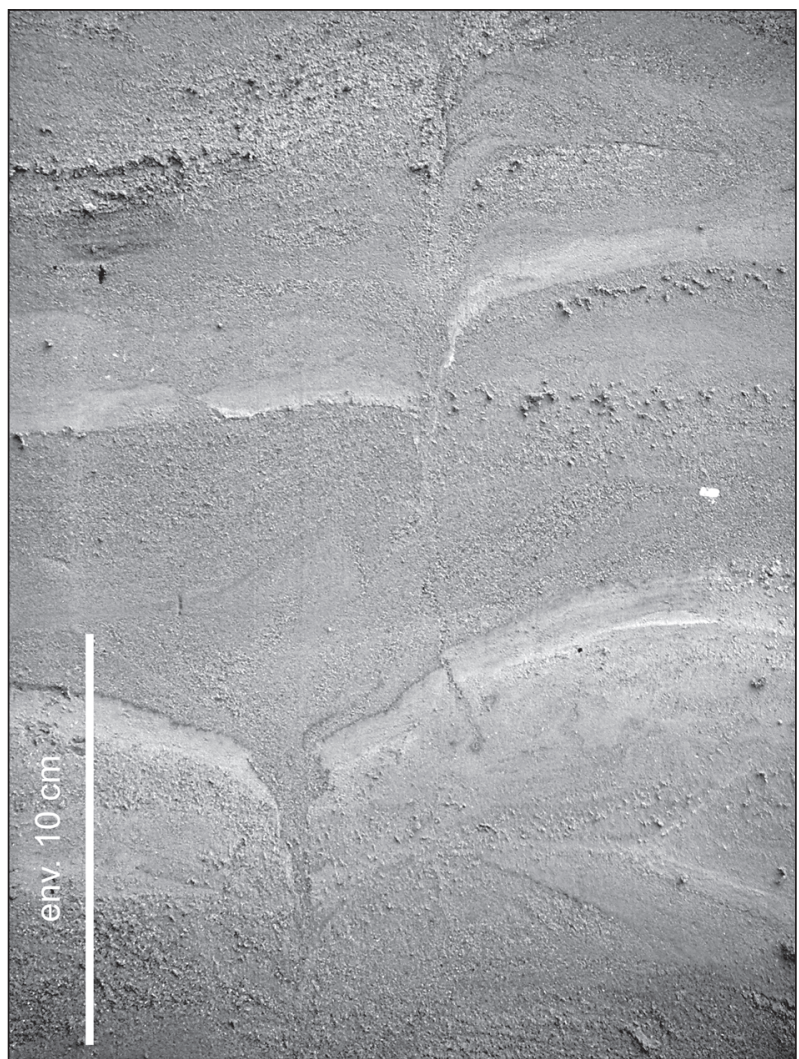

Photo 8: Coupe 1, détail de le la fente de dessiccation syngénétique au sein de l'unité 16.

Photo 8: Cross-section 1, syngenetic frost crack in unit 16.

\subsection{4 - Membre F et F' : limon et limon sablonneux lité}

La partie supérieure des dépôts observés sur la rue Hegel (environ 4 mètres) est constituée de limon sablonneux aux litages discrets (photo 3). La richesse du sédiment en malacofaune et en calcaire (certains lits, très clairs et très calcaires résultent d'une décantation) est variable. Une ligne de fentes de dessiccation est visible à trois mètres de profondeur. Au-dessus de cette ligne, le sédiment est daté par OSL à 23,7 $\pm 2,0 \mathrm{ka}$. Au-dessus de 2,5 mètres de profondeur, les dépôts sont affectés par

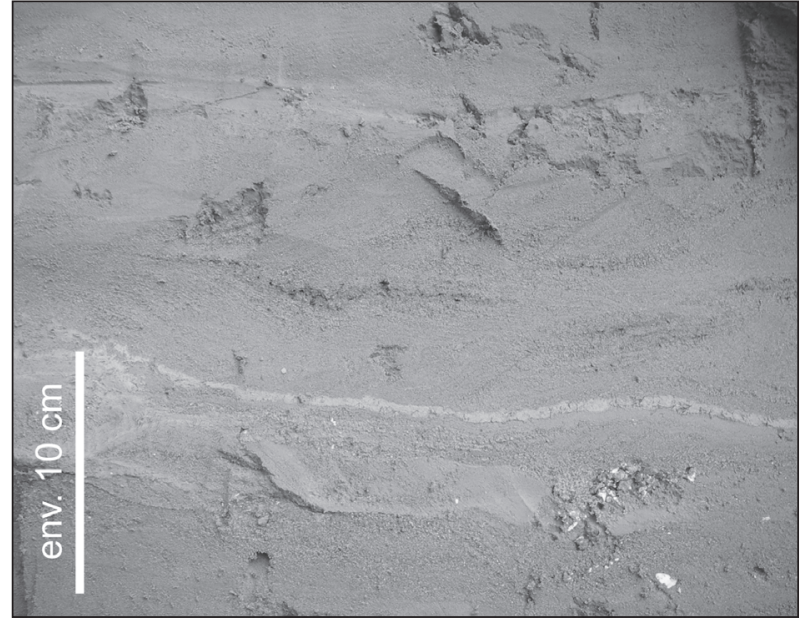

Photo 7: Coupe 1, détail de l'unité 16 et partie supérieure de l'unité 17.

Unité sableuse avec quelques lits de sédiments fins, avec lits entrecroisés et petits dépôts de débris organique (lits sombres).

Photo 7: Cross-section 1, unit 16 detail and upper part of unit 17 . Sandy unit with a few fine sediment beds and cross laminations, organic deposits (dark beds).

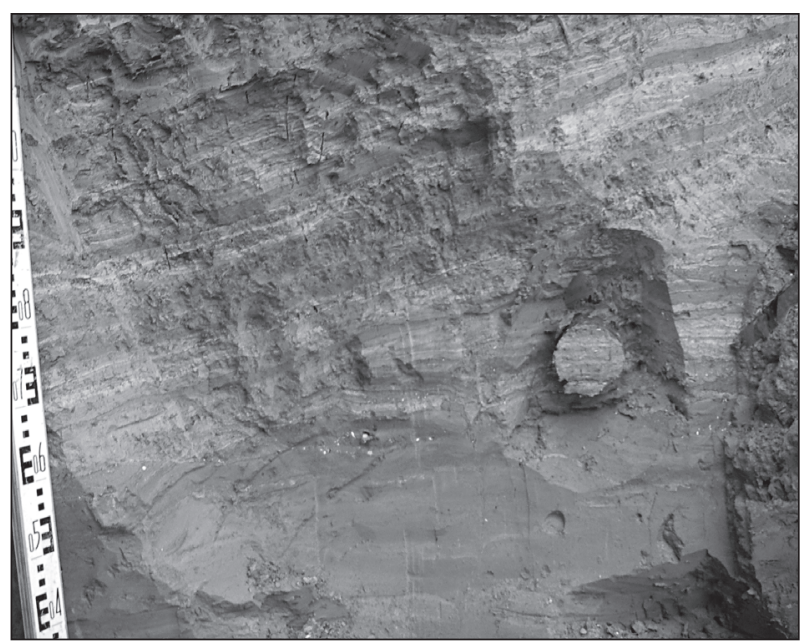

Photo 9: Coupe 1, unité 15 et prélèvement OSL.

L'unité $15 \mathrm{a}$, à gauche, est composée de lits à fort pendage en discordance avec l'unité 15 b. L'ensemble repose sur l'unité 16 , plus sableuse. Photo 9: Cross-section 1, unit15 and OSL sampling. On the left side unit 15 a is made of strongly tilted beds in unconformity with unit $15 \mathrm{~b}$. The whole lies on sandier unit 16

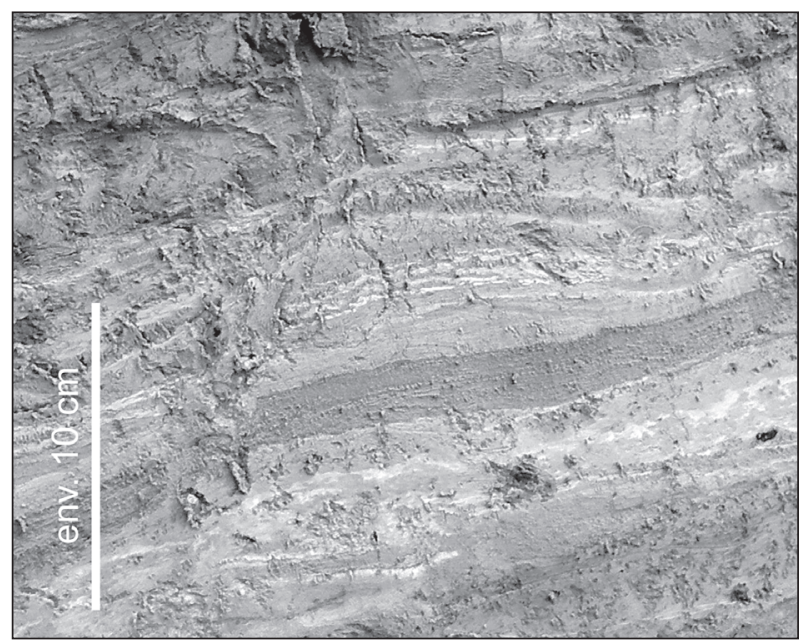

Photo 10: Coupe 1, détail de l'unité 15a.

Lits fins et réguliers d'argile et de sablon.

Photo 10: Cross-section 1, unit 15a detail. Fine and regular clay and sand beds 


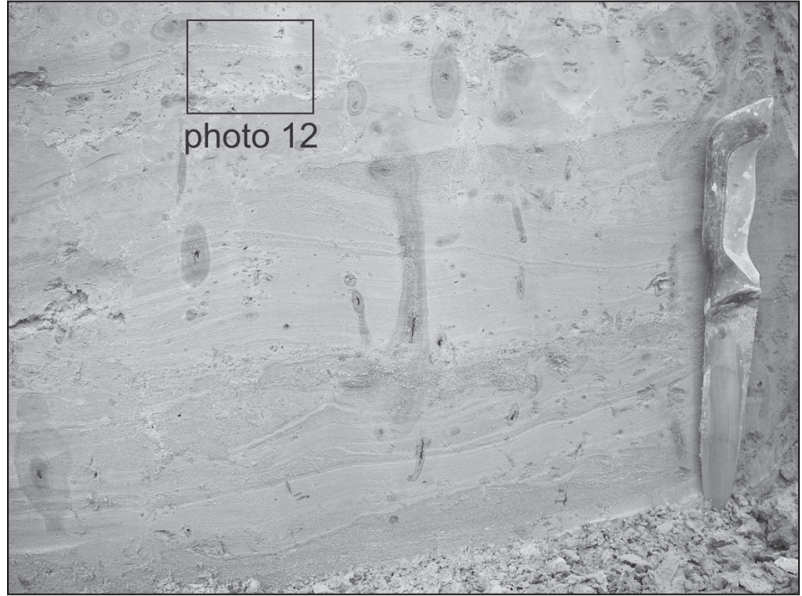

Photo 11 : Coupe 1, détail de l'unité 11b.

Très fin litages de sablon et de quelques lits argileux en petits chenaux interstratifiés; le faciès passe latéralement à un limon lité (11a).

Photo 11: Cross-section 1, unit 11b detail. Very fine sandy beds and few clayed beds in interbedded little channels; the facies laterally shifts to a bedded loam (11a)

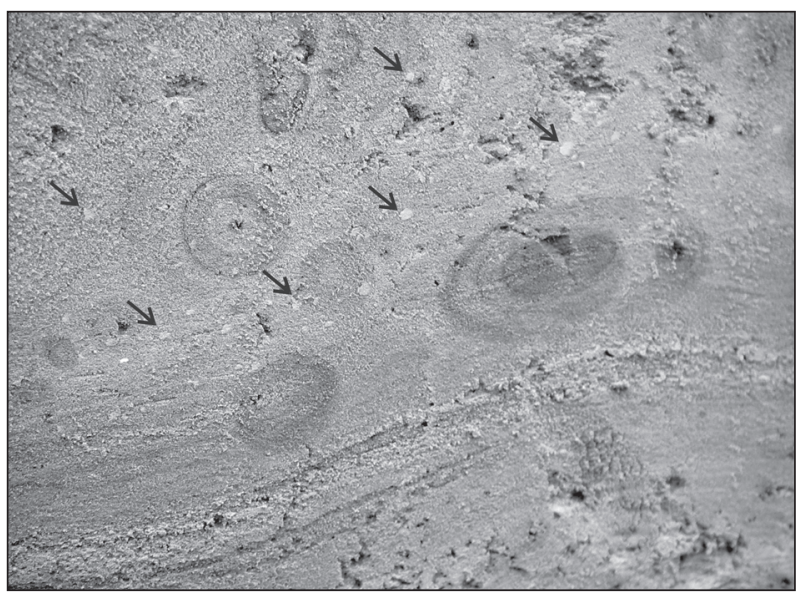

Photo 12: Coupe 1, détail des agrégats de sédiment fin au sein de l'unité 11b.

Quelques-uns sont indiqués par une flèche.

Photo 12: Cross-section 1, fine sediment aggregates details in unit 11b. Arrows point few of them.

la pédogenèse $\left(F^{\prime}\right)$ : le limon prend une coloration brun jaune, est décarbonaté et les litages sont de moins en moins bien perçus.

\section{3 - INTERPRÉTATION ET COMMENTAIRES}

\section{1 - LE PASSAGE D'UNE DYNAMIQUE FLUVIATILE DOMINANTE À UNE DYNAMIQUE ÉOLIENNE DOMINANTE}

Les observations montrent clairement le passage d'une sédimentation fluviatile sableuse (membres $\mathrm{A}$ et $\mathrm{B}$ ) à une sédimentation plus fine, à dominante limoneuse, où les processus éoliens sont de plus en plus prépondérants à la fin du Pléniglaciaire weichselien (membres C à F).

\subsection{1 - La partie inférieure de la séquence}

Le membre A est constitué d'une accumulation majoritairement sableuse en chenaux interstratifiés. La présence, très épisodique d'un sand wedge confirme un assèchement temporaire en conditions périglaciaires rigoureuses. Il est possible (mais non certain) que la base du membre $\mathrm{A}$, observée à partir de 11 mètres d'altitude, débute directement sur la craie 9 mètres plus bas (2 mètres d'altitude) impliquant alors une accumulation sableuse de 12 mètres d'épaisseur. Le membre B est de faciès très proche. Toutefois, la généralisation de très petits chenaux interstratifiés et la présence d'une fente de dessiccation syngénétique semblent indiquer une baisse de la dynamique fluviale. D'ailleurs B constitue le terme de l'accumulation sableuse.

\subsection{2 - La partie supérieure de la séquence}

Le membre F, qui clôt la séquence, est observé dans l'ensemble de la vallée de la Deûle dans la région de Lille. Il se caractérise par la présence de faisceaux ondulés de lamines limoneuses lœssiques alternant avec des lamines de sédiments très fins, voire plastiques, dont la coloration très claire, parfois même blanche, s'explique par une concentration accrue en carbonate. Ce faciès est interprété comment le remaniement par les eaux du limon lœssique présent dans le fond de vallée (dépôt direct dans le fond de vallée, ruissellement diffus depuis les versants) suivi par une décantation (Deschodt et al., 2004, Praud et al., 2007).

\subsection{3 - La partie intermédiaire}

Le passage entre la phase hydrologiquement très active $(\mathrm{A} / \mathrm{B})$ et les accumulations limoneuses à dominante éolienne (F) s'effectue sur environ 1,5 mètre d'épaisseur, entre 14,5 et 16 mètres d'altitude. Tout d'abord, le membre $\mathrm{C}$ semble se déposer en continuité avec la formation sableuse, mais avec une sédimentation essentiellement limono-argileuse mise en place par décantation dans des chenaux. Le membre $\mathrm{D}$, épandage limoneux à gravier de craie épars de petite taille et sans structures perceptibles, a pu être mis en place par accumulation éolienne. Il témoignerait alors d'un arrêt des écoulements, au moins à l'endroit des observations. Une faible dynamique fluviatile reprend avec le dépôt du membre E qui préfigure les accumulations limoneuses régulièrement litées du membre F.

\section{2 - DATATIONS ET COMPARAISONS}

Les datations effectuées rue Hegel font intervenir deux méthodes différentes: une date radiocarbone dans la formation sableuse et deux datations OSL (aliquotes multiples). La calibration de la date radiocarbone nous permet toutefois de les confronter. Nous pouvons également tenter des corrélations à l'échelle de la vallée et de la région.

Les deux principaux pôles de l'évolution du fluviatile vers l'éolien, membre $\mathrm{A} / \mathrm{B}$ d'une part et $\mathrm{F}$ d'autre part, sont une constante rencontrée à l'échelle du fond de vallée (Deschodt et al., 2004). La même évolution 
est rencontrée avec des faciès un peu différents (passage à des sables de couverture éoliens et non à des limons) dans la plaine voisine de la Scarpe (Deschodt et al., 2012) et surtout, plus au nord, dans les larges systèmes de vallées de Belgique, des Pays-Bas et du nord de l'Allemagne (Vandenberghe, 1985; Van Huissteden \& Vandenberghe, 1988; Vandenberghe, 1995; Mol, 1997; Huisink, 2000; Van Huissteden et al., 2000; Kasse et $a l ., 2003,2007)$ où les formations à dominante fluviatile (sables de couverture anciens 1 , Older coversand I) passent aux environs de $17 \mathrm{ka}$ à un niveau de déflation éolienne (Beuningen gravel bed) puis à une accumulation à dominante éolienne (sables de couverture ancien 2, Older coversand II) (Kasse et al., 2007).

Nous ne savons pas si la transition (membres C à E) est généralisable à toute la largeur du fond de vallée ou si elle est liée à une dynamique morphologique locale. Quoi qu'il en soit, la transition entre les dépôts sableux et argileux (passage de $\mathrm{B}$ à $\mathrm{C}$ ) est à cet endroit très rapide comme semble l'indiquer la présence de fentes de gel syngénétiques communes aux deux membres. Rue Hegel, la transition fluviatile/éolien se caractérise aussi par un retour limité à un dépôt fluviatile (membre E; photo 12). A notre connaissance il n'a pas été observé ailleurs de reprise d'activité fluviatile au sein d'une couverture éolienne ou fluvio-éolienne avec éolien dominant. Le membre E pourrait correspondre au dépôt lié à des écoulements sporadiques qui perdurent dans un contexte presque exclusivement éolien à la fin du Pléni- glaciaire. Nous interprétons ainsi la zone un peu plus à l'est où les sondages géotechniques décrivent systématiquement une tendance sableuse (cf. infra, fig. 6 et $\mathrm{n}^{\circ} 10$ sur la figure 7). La présence du membre E rue Hegel pourrait résulter d'une dilatation temporaire de la bande active (épisode plus humide avec une implication paléoclimatique?). Elle pourrait aussi n'être qu'un phénomène local avec un ultime écoulement sur le premier loss de fond de vallée avant une migration définitive plus à l'est. A l'instar du passage $\mathrm{B} / \mathrm{C}$ en limite inférieure, les membres $\mathrm{C}$ à $\mathrm{E}$ représentent probablement une période assez brève.

La datation obtenue à 8,7 mètres de profondeur $(17960$ \pm 90 BP, Lyon-3910-GrA, soit $21639 \pm 361$ cal. BP) est concordante avec les datations récentes obtenues par OSL sur les dépôts fluvio-éoliens (fluvial dominant) de l'Older Coversand I des Pays-Bas (environ 25 à $17 \mathrm{ka}$ ) (Kasse et al., 2007). Les intervalles de confiance sur les dates OSL sont plus importants (fig. 8). Si elle est retenue, la date OSL du membre F $(23,7 \pm 2,0 \mathrm{ka})$ implique une mise en place très rapide (avec un taux de sédimentation de l'ordre de 2 mètres par siècle). L'envahissement lœssique du fond de vallée serait sensible avant $21,7 \mathrm{ka}$ et serait donc contemporain des dépôts fluvio-éoliens de l'Older Coversand I des Pays-Bas et des formations limono-sableuses de la plaine de la Scarpe (datations OSL à $21,6 \pm 2,0$ ka et 20,16 $\pm 1,97 \mathrm{ka}$; Deschodt et al., 2012). La mise en place d'un sédiment éolien en fond de vallée serait donc ici relativement précoce. Elle est

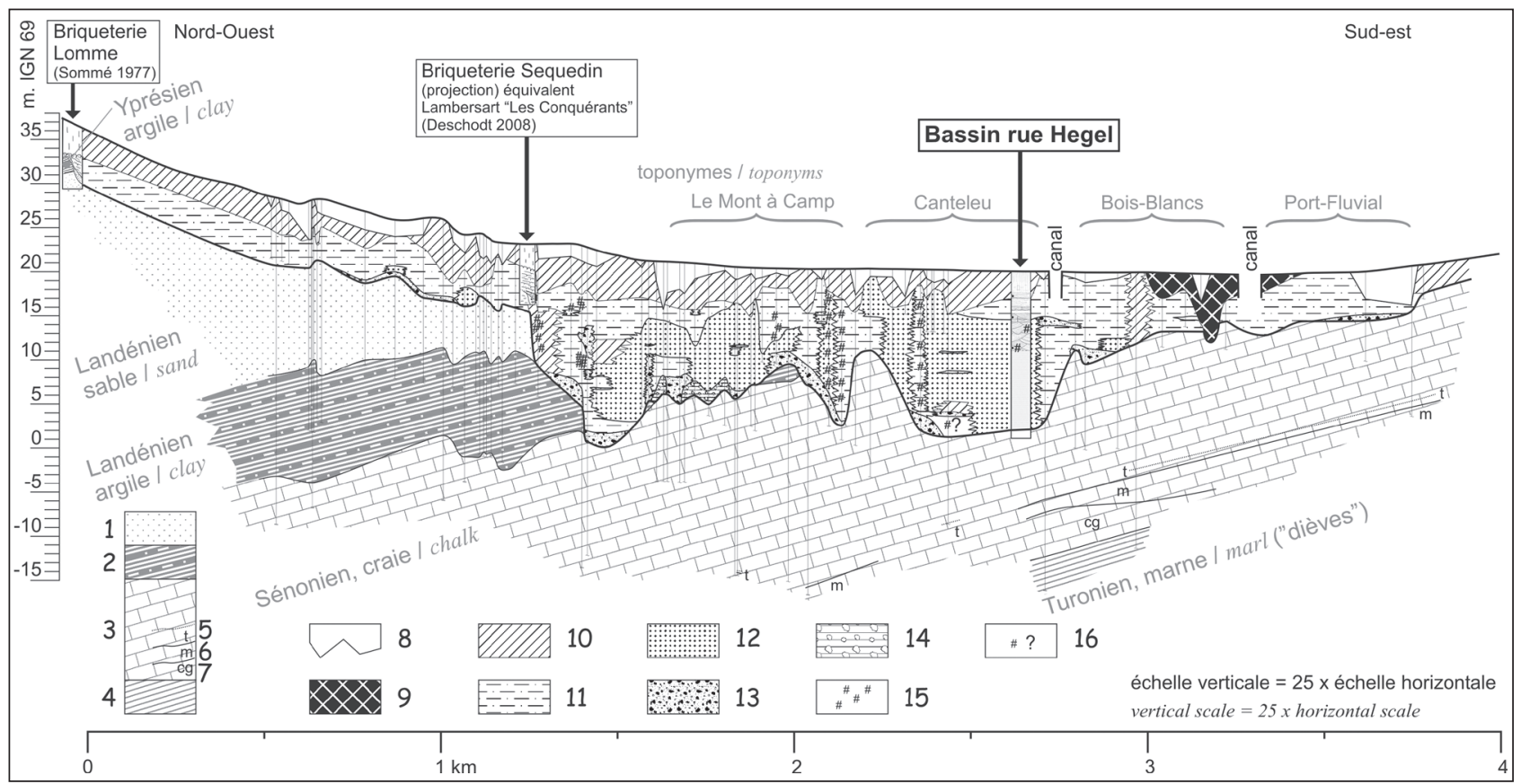

Fig. 6: Transect du versant du Weppes et du fond de vallée de la Deûle passant par la rue Hegel.

1/ Landénien (Eocène), principalement sableux, 2/ Landénien (Eocène), principalement argileux, 3/ craie sénonienne avec niveaux repères, 4/ marne turonienne, 5/ « tun», niveau phosphaté, 6/ « meulière», 7/ « craie grise », 8/ remblais et remaniements, $9 /$ mention de tourbe ou de « vase», 10/ limon (mention d' «argile», de «limon» ou de «silt»), 11/ limon sableux (mention d' «argile» ou de «limon» sableux), 12/ mention de sable (avec éventuelle composante limoneuse), 13/ graviers (craie et/ou silex), 14/ mention d'argile avec gravier de craie, 15/ niveaux organiques (mention «gris organique», «traces organiques», «tendance organique brun foncé», «débris végétaux en veinules», «tourbe»), 16/ mention de «terre noire».

Fig. 6: Weppes slope and Deûle valley bottom cross-section by Hegel street. 1/ Landenian (Eocene), meanly sandy, 2/ Landenian (Eocene), meanly clayed, 3/ senonian chalk with marker beds, 4/ turonian marl, 5/ "tun", phosphatic level, 6/ "meuliere", 7/ "craie grise”, 8/ banks and reworked sediments, 9/ peat or pond mud description, 10/ loam (clay, loam or silt description), 11/ sandy loam (sandy loam or sandy clay description), 12/ sand description (with possible loamy part), 13/ gravels (chalks and/or flint), 14/ clay with chalk gravels description, 15/ organic markers ("organic grey", "organic marks", "dark brown organic trend", "vegetals remains stringlets", "peat"), 16/ "black earth" description. 


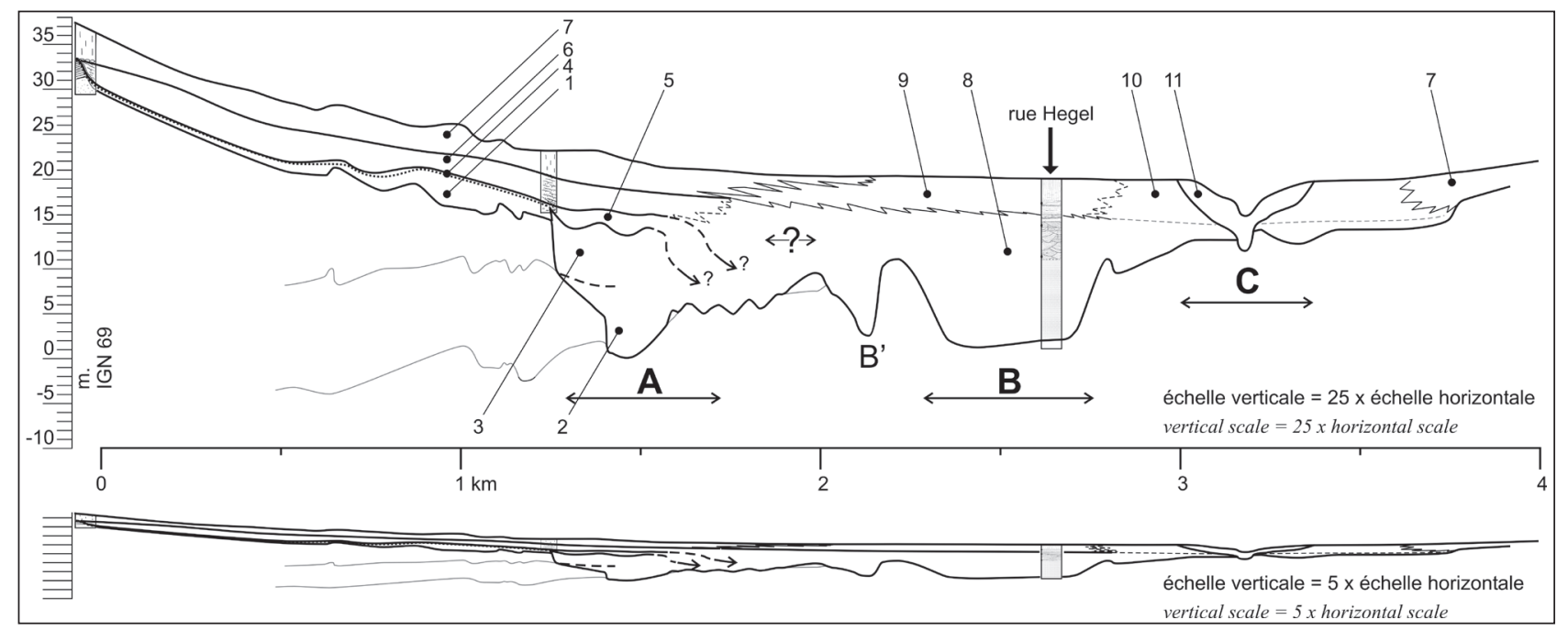

Fig. 7 : Interprétation de la figure 6.

1/ formation de versant limono-sableuse anté-weichselienne, 2/ base du comblement de A; emboîtement de deux nappes? Pléistocène moyen?, 3/ partie supérieure du comblement de A; descriptions de sédiment fin à tendance organique marqué. Début-Glaciaire weichselien (+ Eemien?), 4/ érosion sur versant, formation fluviatile de versant peu épaisse, transition Début-Glaciaire/Pléniglaciare weichselien ou début du Pléniglaciaire weichselien, 5/ prolongation en fond de vallée de 4, formation litée sableuse, transition vers une incision Début-Glaciaire?, 6/ formation de versant limono-sableuse lité, Pléniglaciaire weichselien inférieur et moyen; transition vers une incision contemporaine en fond de vallée?, $7 /$ formation éolienne loessique sur versant, Pléniglaciaire supérieur weichselien, 8/ formation fluviatile sableuse, Pléniglaciaire supérieur, 9/ formation éolienne lœessique en fond de vallée, Pléniglaciaire supérieur weichselien, 10/ formation fluvio-éolienne limono-sableuse en fond de vallée, Pléniglaciaire supérieur weichselien terminal, 11/ formation fluviatile organique, Tardiglaciaire-Holocène. (A) incision et comblement antérieur au Début-Glaciaire weichselien, (B) incision et comblement pléniglaciaire weichselien, (B') probable répétition de la rive gauche du chenal B due à des sondages projetés un peu plus éloignés de l'axe du transect et de la courbure de $\mathrm{B},(\mathrm{C})$ chenal du Tardiglaciaire-Holocène.

Fig. 7: figure 6 interpretation. 1/ ante-Weichselian sandy-loam slope formation, 2/ bottom part of A infilling; cut and fill alluvial sheets? Two alluvial sheets? Middle Pleistocene?, 3/ upper part of A infilling; descriptions of fine sediment with pronounced organic part. Weichselian Early Glacial (+ Eemian?), 4/ slope erosion, slim slope fluvial formation. Weichselian Early Glacial/Pleniglacial transition or Weichselian Pleniglacial start, 5/ unit 4 valley bottom extension, sandy bedded formation, towards an Early Glacial incision?, 6/ bedded sanly-loam slope formation, Weichselian Lower and Middle Weichselian; transition to a bottom valley contemporary incision?, 7/ slope loessic aeolian formation, Upper Pleniglacial, 8/ sandy fluvial formation, Upper Pleniglacial, 9/ bottom valley loessic aeolian formation, Upper Pleniglacial, 10/ bottom valley sandy-loam fluvio-aeolian formation, Late Upper Pleniglacial, 11/ organic fluvial formation, Lateglacial-Holocene. (A) incision and infilling prior to Weichselian Early Glacial, (B) Weichselian Pleniglacial incision and infilling, (B') likely B channel left bank repetition due to farer borings and channel curvature (C) Lateglacial-Holocene channel.

toutefois chronologiquement cohérente avec le début de la mise en place du lœss homogène sur versant (lœss brabantien) (vers 20-22 ka; Haesaerts et al., 1981; van den Haute et al., 1998), postérieur à l'horizon à langues de Nagelbeek.

Ces résultats forment un premier corpus de datations numériques sur le remblaiement de la fin du Pléniglaciaire weichselien dans la plaine de la Deûle. Peu nombreux, ils doivent être considérés avec prudence et nécessitent d'être confortés par d'autres observations et datations.

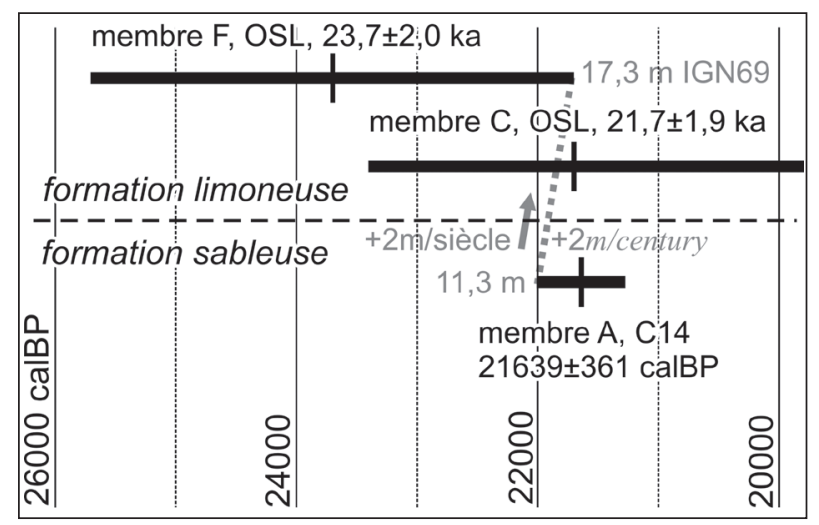

Fig. 8: Expression graphique des datations et taux de sédimentation possible.

Fig. 8: Dating graphic display and possible sedimentation rate.

\section{4 - MISE EN CONTEXTE À L'ÉCHELLE DU VERSANT : INCISIONS, REMBLAIEMENTS ET MIGRATIONS DU COURS AU PLÉISTOCÈNE}

\section{1 - CONSTRUCTION DU TRANSECT}

Les observations de la rue Hegel sont replacées dans le contexte du fond de vallée à travers le transect de la figure 7 dont le point d'origine est l'ancienne briqueterie de Lomme en partie haute du versant (Sommé, 1977). La figure est construite à partir de sondages enregistrés à la banque du sous-sol du BRGM. Nous avons rajouté la projection de l'observation proche de la briqueterie de Sequedin (Sommé, 1977). Ce dernier est considéré comme un équivalent stratigraphique rencontré à Lambersart «Les Conquérants»: en position de base de versant et à proximité de la jonction avec le fond de vallée, des formations alluviales attribuées au DébutGlaciaire weichselien sont scellées par le lœss pléniglaciaire (Deschodt et al., 2008).

L'interprétation d'unités stratigraphiques à travers des descriptions disparates de formations essentiellement sablo-limoneuses est délicate: le même sédiment est décrit de manière différente en fonction des sondeurs. Nous avons donc fait le choix, outre les formations grossières, de conserver trois grandes classes de représentation en fonction de la texture: a) absence de mention de 
sable et description d'une fraction fine («limon», «silt», «argile»), b) mention de sable et de limon, c) mention principale de sable (figurés 10 à 12 de la figure 7).

\section{2 - INTERPRÉTATION ET COMMENTAIRES}

L'incision la plus ancienne se situe sous le bas du versant actuel et atteint la cote 0 (A sur la figure 8). Lérosion fluviatile a exploité la limite structurale entre la craie sénonienne et les premiers dépôts éocènes, l'incision de la craie est modérée. La formation d'argile à graviers de craie $\left(\mathrm{n}^{\circ} 15\right.$ sur la figure 7$)$ conservée en rive droite est probablement un témoin de cette phase de creusement. Des lambeaux d'argile landénienne en place sont conservés encore un peu plus à l'est (et un peu plus haut en vertu du pendage général des couches) vers $\mathrm{x}=$ $2 \mathrm{~km}$. Lincision A dans la craie totalise environ 300 à 400 mètres de large.

Le comblement de A est complexe, avec probablement deux nappes emboîtées à sa base (unité 2 sur la figure 8 ) perçues à travers la présence de deux nappes grossières étagées (fig. 7) et une topographie étagée du toit de la craie. La partie supérieure du comblement (unité 3, fig. 8) correspond à des mentions remarquables de sédiments fins associés à des faciès organiques bruns. Ces dépôts peuvent être corrélés avec les alluvions de fond de vallée du Début-Glaciaire observées dans la même position topographique à Sequedin (Sommé, 1977) et Lambersart «Les Conquérants» (Deschodt et al., 2008). Nous n'excluons pas, toutefois, une possible présence d'alluvions interglaciaires (Eemien).

La fin du comblement de A est postérieure à l'unité 1, formation de versant limono-sableux ante-weichselienne (attribuée au Saalien). Cette formation a subi une érosion aréolaire accompagnée d'un dépôt peu épais de graviers (observés surtout en bas de versant: 4; fig. 8). Cette phase érosive majeure sur versant attribuée à la transition Début-Glaciaire/Pléniglaciaire ou au tout début du Pléniglaciaire (Deschodt et al., 2008) est raccordée à une nappe sableuse fluviatile peu épaisse présente dans le fond de vallée contemporain (5).

La formation lœssique sableuse litée du début du Pléniglaciaire couvre ensuite les anciens chenaux inscrits dans l'incision A. L'accumulation participe à la migration vers le sud-est de la bande active

L'incision $\mathrm{B}$ érode la craie sur près de 500 mètres de large et environ 10 mètres de profondeur, environ 1 kilomètre plus à l'est de l'incision $\mathrm{A}$. L'incision $\mathrm{B}$ ' visible sur le transect est probablement la rive gauche de l'incision B perçue une seconde fois en raison de l'utilisation de sondages un peu plus éloignés. Le transect ne permet pas de proposer une stratigraphie entre ces deux incisions. La datation de l'incision du chenal B est également inconnue. Elle est vraisemblablement postérieure au Début-Glaciaire où le cours était situé nettement plus à l'ouest. Seule la partie supérieure du comblement (unité 8) a été directement observée et datée du Pléniglaciaire (cf. supra). Les sondages géotechniques indiquent une partie inférieure du comblement apparemment homogène et similaire aux formations fluviatiles observées. Nous pouvons émettre l'hypothèse d'une incision au début du Pléniglaciaire supérieur weichselien. Des datations supplémentaires à proximité du bed-rock seraient toutefois nécessaires pour le prouver. Au cours du Weichselien, le versant et le fond de vallée sont recouverts de loess (unités 7 et 9). Si le loess sur versant est un faciès typique, dans le fond de vallée il prend l'aspect d'un loss lité remanié par les eaux (lamines de sédiments fins enrichies en carbonates et ondulées; Deschodt et al., 2004) et intègre une composante sableuse discrète très rarement décrite dans les sondages géotechniques. Le fait nous a incité à individualiser l'unité 10 , encore un peu plus à l'est où, au contraire, tous les sondages font mention d'une composante sableuse. Il s'agit probablement d'une zone, large d'environ 700 à 800 mètres, où quelques écoulements perdurent à la fin du Pléniglaciaire supérieur weichselien. La discrète morphologie fluviale associée a pu guider le tracé du chenal tardiglaciaire-holocène $(\mathrm{C})$ qui se développe au sein de cette zone (Deschodt et al. 2004, Deschodt \& Salvador, 2009).

\section{5 - CONCLUSION}

Pendant le Pléniglaciaire supérieur weichselien:

- la Deûle est composée de multiples chenaux peu profonds dans une large bande active;

- la bande active se situe environ 700 mètres plus à l'est que le cours Début-Glaciaire qui est scellé par des dépôts lœssiques de versant;

- l'écoulement pléniglaciaire supérieur (une date ${ }^{14} \mathrm{C}$ à $17960 \pm 90 \mathrm{BP}$, soit $21639 \pm 361 \mathrm{cal}$. BP) finit de combler une incision développée dans la craie;

- en partie supérieure du comblement, la nature des dépôts change rapidement et sans hiatus apparent, avec d'abord des formations litées argileuses (une date OSL à 21,7 $\pm 1,9 \mathrm{ka}$ ), puis des formations lœssiques avec des traces d'écoulements de plus en plus ténues (une date OSL à $23,7 \pm 2,0 \mathrm{ka}$ ).

Ces différentes données permettent d'envisager un changement de l'hydrologie aux environs de 21,7 ka, avec un passage rapide mais apparemment sans hiatus entre un dépôt fluviatile à dominante sableuse à une formation éolienne lœssique. Cette date est en concordance avec le début du dépôt de la couverture lœssique homogène de la fin du Pléniglaciaire supérieur. Elle est plus précoce que la mise en place des dépôts éoliens de l'Older coversand $I$ en zone sableuse. Ces premières datations effectuées sur les comblements pléniglaciaires terminaux des fonds de vallée de la zone loessique sont à conforter.

\section{REMERCIEMENTS}

Je remercie Erik Pearthree pour la relecture de l'abstract, Cyril Castanet et Pierre Antoine pour leurs conseils avisés.

La rédaction de cet article a bénéficié de quelques jours accordés par l'INRAP Nord-Picardie, que je remercie pour la confiance qui m'a été accordée. 


\section{BIBLIOGRAPHIE}

CERCY C., 2004 - Marquette-lez-Lille, «Le Haut-Touquet». Document final de synthèse, INRAP \& Service Régional de l'Archéologie du Nord-Pas-de-Calais, Villeneuve-d'Ascq, 69 p.

DESCHODT L., SALVADOR P.-G. \& BOULEN M., 2004 - Formations sédimentaires et évolution de la vallée de la Deûle depuis le Pléniglaciaire supérieur à Houplin-Ancoisne (Nord de la France). Quaternaire, 15 (3), 269-284.

DESCHODT L., MUNAUT A.-V., LIMONDIN-LOZOUET N. \& MURIEL B., 2008 - Lambersart «Les Conquérants» (vallée de la Deûle, Nord de la France): une transition versant-fond de vallée au début glaciaire et Pléniglaciaire weichselien. Quaternaire, 19 (4), 309-333.

DESCHODT L., BOULEN M. \& PRAUD I., 2008 - Le Marais de Santes : évolution de la Deûle en rapport avec l'occupation humaine. Archéopages, 23, 22-27.

DESCHODT L. \& SALVADOR P.-G., 2009 - L'évolution de la vallée de la Marque dans le secteur de Bouvines (Nord, 59) depuis la fin du Pléniglaciaire weichselien. Revue du Nord, 91 (383), 19-27.

DESCHODT L., SALVADOR P.-G., FERAY P. \& SCHWENNINGER J.-L., 2012 - Transect partiel de la plaine de la Scarpe (Bassin de 1'Escaut, nord de la France). Stratigraphie et évolution paléogéographique. Quaternaire, 23 (1), 87-116.

HAESAERTS P., JUVIGNÉ E., KUYL O., MÜCHER H. \& ROEBROEKS W., 1981 - Compte rendu de l'excursion du 13 juin 1981, en Hesbaye et au Limbourg Néerlandais, consacrée à la chronostratigraphie des lœss du Pléistocène supérieur. Annales de la Société Géologique de Belgique, 104, 223-240.

HUISINK M., 2000 - Changing River styles in response to weichselian climate changes in the Vecht valley, eastern Netherlands. Sedimentary Geology, 133 (1-2), 115-134.

KASSE C., VANDENBERGHE J., VAN HUISSTEDEN J., BOHNCKE S.J.P. \& BOS J.A.A., 2003 - Sensitivity of Weichselian fluvial systems to climate change (Nochten mine, eastern Germany). Quaternary Science Reviews, 22 (20), 2141-2156.

KASSE C., VANDENBERGHE D., DE CORTE F. \& VAN DEN HAUTE P., 2007 - Late Weichselian fluvio-aeolian sands and coversands of the type locality Grubbenvorst (southern Netherlands): sedimentary environnements, climate record and age. Journal of Quaternary Science, 22 (7), 695-708.

LADRIÈRE J., 1883 - Le Terrain quaternaire du fort du Vert-Galant, comparé à celui des régions voisines. Annales de la Société Géologique du Nord, 1882-1883, 10, 86-99.

LADRIÈRE J., 1885 - Compte-rendu de l'excursion de la Société géologique du Nord aux environs de Lille et considérations sur les terrains quaternaires et récents des vallées de la Lys et de la Deûle. Annales de la Société Géologique du Nord, 1884-1885, 12, 405-422.
MOL J., 1997 - Fluvial response to weichselian climate changes in the Niederlausitz (Germany). Journal of Quaternary Science, 12 (1), 43-60.

PRAUD I., BERNARD V., BOITARD E., BOSSUT D., BOULEN M., BRAGUIER S., CLARYS B., COUBRAY S., CRETEUR Y., DELAGNE F., DESCHODT L., DIETSCH-SELLAMI M.-F, FABRE J, FECHNER K, JOUANIN G, LANTOINE J., LEMOULANT Q., MAIGROT Y., MARTIAL E., MICHEL L., MONCHABLON C., PALAU R., PONE, P. \& VAN DER PLICHT J., 2007 - Houplin-Ancoisne (59), «Le marais de Santes ». Rapport de fouille archéologique. Service Régional de l'Archéologie du Nord-Pas-de-Calais, Villeneuve-d'Ascq, 02/0227/FOU, GB6115021001, 2 tomes, 290 pages.

SALVADOR P.-G., DESCHODT L. \& COUTARD S., 2005 Nouvelles observations sur la stratigraphie des formations holocènes (récent) de la Plaine de la Lys à Houplines (Nord). Quaternaire, 16 (1), 65-68.

SOMMÉ J., 1968 - Coupe dans le Quaternaire récent de la vallée de la Deûle, près de Lille. Bulletin de l'Association Française pour l'Étude du Quaternaire, 5 (2), 89-99.

SOMMÉ J., 1977 - Les plaines du Nord de la France et leur bordure: étude géomorphologique. Thèse de Doctorat d'État, Université Paris 1 Panthéon-Sorbonne, Paris, 2 vol., 810 p.

TUFFREAU A., RÉVILLION S., SOMMÉ J. \& VAN VLIETLANOË B., 1994 - Le gisement paléolithique moyen de Seclin (Nord). Bulletin de la Société Préhistorique Française, 91 (1), 23-46.

VANDENBERGHE J., 1985 - Paleoenvironment and stratigraphy during the Last Glacial in the belgian-dutch border region. Quaternary Research, 24 (1), 23-38

VANDENBERGHE J., 1995 - Timescales, climate and river development. Quaternary Science Reviews, 14 (6), 631-638.

VAN DEN HAUTE P., VANCRAEYNEST L. \& DE CORTE F. 1998 - The Late Pleistocene loess deposits and palaeosols of eastern Belgium: new TL age determinations. Journal of Quaternary Science, 13 (5), 487-497.

VAN HUISSTEDEN J. \& VANDENBERGHE J., 1988 - Changing fluvial style of periglacial lowland rivers during the Weichselian Pleniglacial in the eastern Netherlands. Zeitschrift für Geomorphologie, Neue Folge, Suppl. 71, 131-146.

VAN HUISSTEDEN J., VANDENBERGHE J., VAN DER HAMMEN T. \& LAAN W., 2000 - Fluvial and aeolian interaction under permafrost conditions: Weichselian Late Pleniglacial, Twente eastern Netherlands. Catena, 40 (3), 307-321.

WENINGER B. \& JÖRIS O., 2008 - A ${ }^{14} \mathrm{C}$ age calibration curve for the last $60 \mathrm{ka}$ : the Greenland-Hulu U/Th timescale and its impact on understanding the Middle to Upper Paleolithic transition in Western Eurasia. Journal of Human Evolution, 55 (5), 772-781. 